\title{
FTO genotype is associated with body mass index and waist circumference in Mexican young adults
}

\author{
Margarita Teran-Garcia ${ }^{1}$, Itzel Vazquez-Vidal ${ }^{1}$, Flavia C. D. Andrade ${ }^{2}$, Michelle Mosley ${ }^{1}$, \\ Eduardo Medina-Cerda ${ }^{3}$, Celia Aradillas-Garcia ${ }^{4}$ \\ ${ }^{1}$ Department of Food Science and Human Nutrition, University of Illinois, Champaign, USA \\ ${ }^{2}$ Department of Kinesiology and Community Health, University of Illinois, Champaign, USA \\ ${ }^{3}$ Health Center, University Autonomous of San Luis Potosí, San Luis Potosí, Mexico \\ ${ }^{4}$ School of Medicine, University Autonomous of San Luis Potosí, San Luis Potosí, Mexico \\ Email: teranmd@illinois.edu
}

Received 20 January 2013; revised 26 February 2013; accepted 8 March 2013

\begin{abstract}
Individual variations in the fat mass and obesityassociated (FTO) gene have been associated with obesity and BMI in diverse populations, but there are no reports in young Mexicans. We explored the association of a common FTO single-nucleotide polymorphism (SNP, rs805704) with obesity-related phenotypes in Mexican young adults. The FTO-SNP was genotyped using the fluorescent polarization method in college-age, apparently healthy subjects from the "UP AMIGOS" cohort (n = 251, 18 - 25 yrs). Homozygotes for the $A$ allele $(15 \%, n=38)$ were heavier $\left(1.6 \mathrm{~kg} / \mathrm{m}^{2}\right.$ and $\left.6.2 \mathrm{~kg}\right)$ and had a larger waist circumference (WC, $4.8 \mathrm{~cm}$ ) than G allele carriers. The FTO genotype was associated with BMI, weight and WC independently of age and sex and explained 2.7 to $3.1 \%$ of the variance in obesity-related phenotypes. The FTO genotype was also associated with fasting glucose $(P=\mathbf{0 . 0 2 8 3})$. No other associations were found in the additive model. Despite our small sample size, we found that the FTO-rs805704 genotype influences obesity-related phenotypes young Mexicans. Previously observed FTO associations with fasting glucose were replicated. Previously reported associations with other metabolic traits likely represent the long-term consequences of obesity.
\end{abstract}

Keywords: FTO; Obesity; Genetic Risk; Young; Mexicans

\section{INTRODUCTION}

The fat mass and obesity-associated (FTO) gene has been identified as a strong candidate for obesity-related phenotypes in several populations, mainly Europeans [1] and Asians, as recently shown in a meta-analysis [2]. However, there are population differences in single nucleotide polymorphisms (SNPs) and their association strength related to obesity-traits, dietary intake or the impact of physical activity $[3,4]$.

A small number of FTO-variants associated with obesity-risk traits are concurrent in all populations and differences in minor allele frequency (MAF) or linkage disequilibrium (LD) patterns may explain divergences in the strength of those associations [2]. Still, most reports agree that markers in or nearby the FTO gene explain only $1 \%$ to $2 \%$ of the variability in BMI and each FTO risk allele increases BMI by 0.26 to 0.66 units $\left(\mathrm{kg} / \mathrm{m}^{2}\right)$, and the odds of being obese by $\sim 1.3[2,5]$. To date, many of the associations reported with obesity-risk are located in the cluster that includes the first and second intron of the FTO gene $[2,6]$. Others have found that FTO-variants may impact satiety, eating behaviors or measures of energy intake and expenditure $[4,7]$. FTO-variants also have been associated with glucose homeostasis and diabetes $[6,8,9]$.

Replications of FTO markers and meta-analysis are available for large cohorts of Caucasian, Asian and African-Americans, but few reports include MexicanAmerican, Mexican or Amerindian populations [9-11]. Reports from Mexicans and Mexican-Americans include mainly adults already diagnosed with diabetes, obesity or obesity-related diseases $[10,11]$. In the quest to understand the genetic architecture of complex diseases, it is important to report allele frequencies and both positive and negative associations on understudied populations.

Our objective is to report the allele frequency and associations of a FTO-variant (rs8057044) with obesityrelated measures in Mexican young adults.

\section{DESIGN AND METHODS}

\subsection{Population Characteristics}

Participants aged 18 to 25 years belong to the 2009 study cohort "UP AMIGOS" (Universities of San Luis Potosí 
and Illinois: A Multidisciplinary Investigation on Genetics, Obesity, and Social-Environment). The UP AMIGOS study has been described previously [12]. In this report, observations from a subset of those with signed inform consent who also donated blood samples remnants for genetic studies was analyzed. The protocol was reviewed and approved by the Institutional Review Boards at Universidad Autónoma de San Luis Potosí and University of Illinois Champaign-Urbana.

\subsection{Protocol}

Following an overnight fast ( $\geq 8$ hours) and a blood draw for biomarkers, all participants underwent a health screening at the university clinic. The screening consisted of: a) anthropometric measurements and blood pressure; b) physical exam and medical interview.

\subsection{Health Screening}

Height was measured and recorded to the nearest centimeter and weight was recorded to the nearest $0.1 \mathrm{~kg}$. International standards were used to classify individuals as normal weight, overweight or obese according to their BMI in $\mathrm{kg} / \mathrm{m}^{2}$ [13]. Waist circumference (WC) was measured using a flexible, non-stretching tape at the level of the iliac crest following normal expiration with the subject in standing position and was recorded to the nearest $0.1 \mathrm{~cm}$. Abdominal obesity was defined as $\mathrm{WC} \geq$ $90 \mathrm{~cm}$ in males and $\geq 80 \mathrm{~cm}$ in females [13]. Blood pressure was taken on the dominant arm (right, in most cases) while participant was seated using appropriately sized cuffs by trained health care providers.

\subsection{Blood Biomarkers}

Glucose determinations. Blood samples were collected by trained personnel. All samples were measured in the
Alcyon 300 autoanalyzer (Abbott, Illinois, USA, with reagents from BioSystems, Mexico). Fasting glucose (FG) was determined according to the glucose oxidase peroxidase method. Equipment was regularly calibrated by standardized reference samples.

DNA extraction and genotyping. Genomic DNA was extracted from whole blood in 251 samples available (99 men and 152 women) using a commercial DNA extraction procedure (Gentra Puregene Blood Kits, Quiagen, CA, USA) and the FTO SNP rs8057044 was genotyped by template-directed dye-terminator incorporation with the fluorescence polarization detection method, as described by Kwok [14]. Fluorescent signals were read by the VICTOR5 Multi-label Plate Reader (Perkin Elmer Life Sciences, Massachusetts, USA). Genotypes were called by automated software, and independently verified by investigators without discordance. For accuracy of genotypes, we repeated at least $10 \%$ of samples randomly including both positive and negative controls. Concordance for replicates was $99 \%$.

\subsection{Statistical Analysis}

Association was tested for general and additive genotype effect (recessive model, rM; additive model, AM) on all obesity-related traits investigated with general linear models (GLM) using SAS 9.2 (SAS Institute Inc., NC, USA). All models were adjusted for age and sex as covariates. Allele frequencies were estimated by the gene counting method, and the chi-square test was used to identify significant departures from Hardy-Weinberg equilibrium.

\section{RESULTS}

A summary of results and allele frequencies is presented in Table 1. The rs8057044 was in Hardy-Weinberg

Table 1. Population characteristics according to genotypes of the FTO-variant rs8057044.

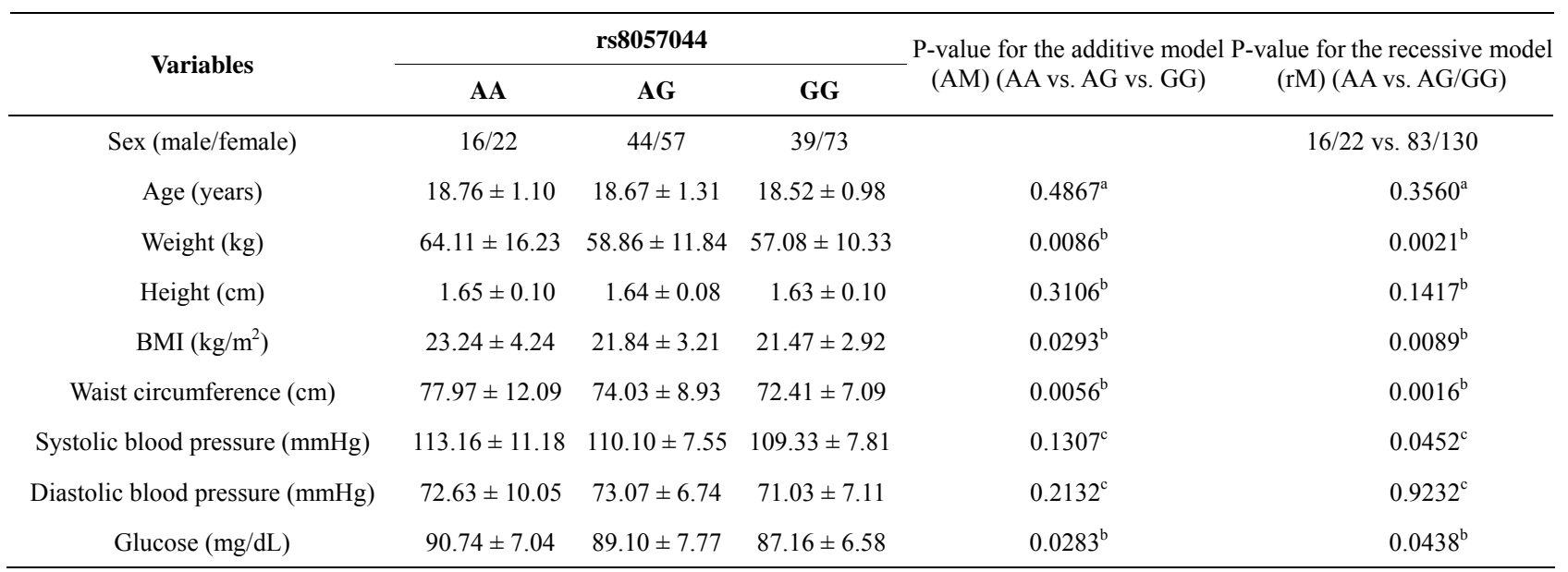

Data are means \pm standard deviation. ${ }^{\mathrm{a}}$ Kruskal-Wallis test; ${ }^{\mathrm{b}}$ Model adjusted for age and sex; ${ }^{\mathrm{c}}$ Model adjusted for age, sex and height. 
equilibrium with a minor allele frequency (MAF) of 0.35 . Individuals with the AA genotype (15.1\%) were heavier $(\mathrm{P}=0.0086$ under the $\mathrm{AM}$ and $\mathrm{P}=0.0021$ under the $\mathrm{rM})$, and had larger WC $(\mathrm{P}=0.0056$ under the $\mathrm{AM}$ and $\mathrm{P}=$ 0.0016 under the rM) than those with other genotypes. Those differences were also consistent with the BMI phenotype $(\mathrm{P}=0.0293$ under the $\mathrm{AM}$ and $\mathrm{P}=0.0089$ under the $\mathrm{rM}$ ).

The FTO genotype explained $2.7 \%, 3.1 \%$ and $2.7 \%$ of the variance in the obesity-related phenotypes investigated (weight, WC and BMI, respectively). A/A homozygotes had higher fasting glucose levels $(\mathrm{P}=0.0283$ under the $\mathrm{AM}$ and $\mathrm{P}=0.0438$ under the $\mathrm{rM}$ ) compared to other genotypes, but still within the normal range. No significant associations with blood pressure or height were found in the AM, but there were statistical differences in systolic blood pressure under the $\mathrm{rM}(\mathrm{P}=$ 0.0452).

\section{CONCLUSIONS}

The main objective of this study was to report the allele frequency and associations of a common marker in the FTO gene with obesity-related phenotypes in young Mexican individuals. There are few reports of FTO markers in Mexican or Mexican-Americans and most include adult individuals with extreme obesity and associated morbidities $[9,10]$.

Data on the modifying effects of FTO gene variants on adiposity in children, adolescents and young adults (18 to 25 year-old) is still scarce. In our study, those with the A/A genotype were heavier, had higher BMI and larger WC compared to those with the other genotypes, independently of sex. Those phenotypic differences are even larger when contrasting AA homozygotes with GG homozygotes for weight, BMI and WC $(7.0 \mathrm{~kg}, 1.77$ $\mathrm{kg} / \mathrm{m}^{2}$, and $5.6 \mathrm{~cm}$, respectively).

Despite the limitations of our sample size, the overall effect we observed of FTO-rs8057044 variant is larger than effect-sizes observed reported for BMI ( 0.26 to 0.66 $\left.\mathrm{kg} / \mathrm{m}^{2}\right)$ and WC $(\sim 2 \mathrm{~cm})$ in Hispanics, Asians or Europeans $[1,2,4,5]$ and in contrast with the null associations with BMI or obesity reported by others $[9$, 15,16]. It is not known if FTO-variants influence weight gain trajectories in Mexicans. However, in Europeans the influence of FTO-variants on weight gain during childhood and adolescent seems to decline by adulthood [17, $18]$.

The FTO gene has been associated with diabetic risk mainly by its effects on BMI [1] and associations with fasting glucose or insulin levels have mainly targeted adult populations $[6,8,9]$. It is possible that observed associations of FTO-variants with diabetes or other metabolic traits represent the long term consequences of obesity. In our sample, FTO-rs8057044 variant was associated with fasting glucose levels $(\mathrm{P}=0.0283$, under additive model) but the overall mean glucose levels were within normal values. Recently, a $\mathrm{CpG}$ site located in the first intron of the FTO gene was shown to be hypomethylated in young individuals that progressed to become diabetic [19]. More longitudinal studies are necessary to investigate if population-specific FTOvariants are associated with FTO-methylation state and lead to deleterious metabolic changes.

The FTO-variant investigated (rs8057044) is highly correlated with other FTO SNPs located in intron $1\left(\mathrm{r}^{2}\right.$ ranging from 0.7 to 0.9 ) in Hispanic-Americans of the IRAS study but, according to their report, moderately correlated with commonly identified SNPs in nonHispanic Whites $\left(\mathrm{r}^{2} 0.4\right.$ to 0.7) [9]. We found similar MAF, magnitude and direction of obesity-associated effects as in the IRAS study [9].

Recent interest on understanding the impact of geneenvironment interactions for obesity prevention has prompted large genetic epidemiological studies to investigate the impact of diet or physical activity. Whereas some reports indicate that lifestyle factors modify the genetic risk of FTO on obesity phenotypes among sedentary individuals with larger calorie intake [20] or that physical activity attenuates the risk of having a large BMI [3], others suggest that having a healthy lifestyle alone is not sufficient to decrease the predisposition for a larger BMI or WC [21].

Longitudinal studies designed to identify which lifestyle factors may act as effect modifiers for obesity risk linked to gene variants are necessary, especially with the current obesogenic environment that enables sedentary behaviors and unhealthy eating. A report on elderly university alumni from Spain, the SUN study, found that FTO associations with obesity-risk might depend on high carbohydrate intake [22]. If we take into account the BMI of study participants when they are young for genetic association analyses, we could begin to understand the accumulative effect of lifestyle events.

In summary, this is the first report of a FTO-variant association with measures of adiposity in apparently healthy Mexican young adults. It is possible that we were able to identify a positive association between FTO and obesity-related phenotypes because we studied young individuals who are yet to be influenced by the accumulative effects of the environment. As individuals enter adulthood, the genetic effect might decline. This was a candidate gene-marker study, so ancestry informative markers are not available at this time. Further studies are required to validate gene-environment associations describing longitudinal changes in age-specific groups and the impact of lifestyle interventions in diverse populations. 


\section{ACKNOWLEDGEMENTS}

Data presented here are a subproject of the UP AMIGOS study group (Universities of San Luis Potosi and Illinois a Multidisciplinary Investigation on Genetics, Obesity and Social-environment). We are grateful to all applicants, medical personnel, and nurses participating in the 2009 data collection. The 2009 study group include: University of Illinois at Urbana-Champaign, USA: F.C.D. Andrade, M. Raffaelli, M. Teran-Garcia, A. R. Wiley; Universidad Autónoma de San Luis Potosí (UASLP), Mexico: C. Aradillas-García, E. de la Cruz-Mendoza, E. Medina-Cerda, D. P. Portales-Pérez, C. Rojas, O. Sánchez-Armáss, S. Rosales Mendoza, J. M. Vargas-Morales; and Instituto Mexicano Del Seguro Social in Durango, Mexico: F. Guerrero-Romero (scientific adviser), M. Rodríguez-Moran, and L. E. Simental-Mendia. This study was funded by 1) the UASLP, Hormones Laboratory at the School of Medicine, the Clinical Biochemistry Laboratory at the Chemical Sciences School, and the UASLP University Health Center under agreement support C09-PIFI-030606 (to Aradillas-Garcia) and 2) the University of Illinois at Urbana-Champaign Research Board grant \#09070 (to Andrade); Center for Health and Aging (to Andrade); and the USDA National Institute of Food and Agriculture, Hatch Projects \#600108-793000-793323 (to Raffaelli) and \#600109-698000-698354 (to Teran-Garcia).

\section{REFERENCES}

[1] Frayling, T.M., et al. (2007) A common variant in the FTO gene is associated with body mass index and predisposes to childhood and adult obesity. Science, 316, 889-894. doi:10.1126/science.1141634

[2] Peng, S., Zhu, Y., Xu, F., Ren, X., Li, X. and Lai, M. (2011) FTO gene polymorphisms and obesity risk: A meta-analysis. BMC Medicine, 9, 71. doi:10.1186/1741-7015-9-71

[3] Kilpelainen, T.O., et al. (2011) Physical activity attenuates the influence of FTO variants on obesity risk: A metaanalysis of 218,166 adults and 19,268 children. PLoS Medicine, 8, e1001116. doi:10.1371/journal.pmed.1001116

[4] Lear, S.A., Deng, W.Q., Pare, G., Sulistyoningrum, D.C., Loos, R.J. and Devlin, A. (2011) Associations of the FTO rs9939609 variant with discrete body fat depots and dietary intake in a multi-ethnic cohort. Genetics Research, 93, 419-426. doi:10.1017/S001667231100036X

[5] Loos, R.J. (2009) Recent progress in the genetics of common obesity. British Journal of Clinical Pharmacology, 68, 811-829. doi:10.1111/j.1365-2125.2009.03523.x

[6] Li, H., et al. (2011) Association of genetic variation in FTO with risk of obesity and type 2 diabetes with data from 96,551 East and South Asians. Diabetologia, 55, 981-995. doi:10.1007/s00125-011-2370-7

[7] Wardle, J., Carnell, S., Haworth, C.M., Farooqi, I.S., O'Rahilly, S. and Plomin, R. (2008) Obesity associated genetic variation in FTO is associated with diminished satiety. The Journal of Clinical Endocrinology \& Metabolism, 93, 3640-3643. doi:10.1210/jc.2008-0472

[8] Hertel, J.K., et al. (2011) FTO, type 2 diabetes, and weight gain throughout adult life: A meta-analysis of 41,504 subjects from the Scandinavian HUNT, MDC, and MPP studies. Diabetes, 60, 1637-1644. doi:10.2337/db10-1340

[9] Wing, M.R., et al. (2011) Analysis of FTO gene variants with obesity and glucose homeostasis measures in the multiethnic Insulin Resistance Atherosclerosis Study cohort. International Journal of Obesity, 35, 1173-1182. doi:10.1038/ijo.2010.244

[10] Villalobos-Comparan, M., et al. (2008) The FTO gene is associated with adulthood obesity in the Mexican population. Obesity, 16, 2296-2301. doi: $10.1038 /$ oby. 2008.367

[11] Jowett, J.B., et al. (2010) Genetic variation at the FTO locus influences RBL2 gene expression. Diabetes, 59, 726-732. doi:10.2337/db09-1277

[12] Andrade, F.C., Raffaelli, M., Teran-Garcia, M., Jerman, J.A. and Garcia, C.A. (2011) Weight status misperception among Mexican young adults. Body Image, 9, 184-188. doi:10.1016/j.bodyim.2011.10.006

[13] Alberti, K.G., et al. (2009) Harmonizing the metabolic syndrome: A joint interim statement of the International Diabetes Federation Task Force on Epidemiology and Prevention; National Heart, Lung, and Blood Institute; American Heart Association; World Heart Federation; International Atherosclerosis Society; and International Association for the Study of Obesity. Circulation, 120, 1640-1645. doi:10.1161/CIRCULATIONAHA.109.192644

[14] Kwok, P.Y. (2002) SNP genotyping with fluorescence polarization detection. Human Mutation, 19, 315-323. doi:10.1002/humu. 10058

[15] Jacobsson, J.A., Riserus, U., Axelsson, T., Lannfelt, L., Schioth, H.B. and Fredriksson, R. (2009) The common FTO variant rs9939609 is not associated with BMI in a longitudinal study on a cohort of Swedish men born 1920-1924. BMC Medical Genetics, 10, 131. doi:10.1186/1471-2350-10-131

[16] Li, H., Wu, Y., Loos, R.J., Hu, F.B., Liu, Y., Wang, J., Yu, Z. and Lin, X. (2008) Variants in the fat mass- and obesity-associated (FTO) gene are not associated with obesity in a Chinese Han population. Diabetes, 57, 264268. doi: $10.2337 / \mathrm{db} 07-1130$

[17] Hardy, R., Wills, A.K., Wong, A., Elks, C.E., Wareham, N.J., Loos, R.J., Kuh, D. and Ong, K.K. (2009) Life course variations in the associations between FTO and MC4R gene variants and body size. Human Molecular Genetics, 19, 545-552. doi:10.1093/hmg/ddp504

[18] Jess, T., et al. (2008) Impact on weight dynamics and general growth of the common FTO rs9939609: A longitudinal Danish cohort study. International Journal of Obesity, 32, 1388-1394. doi:10.1038/ijo.2008.110

[19] Toperoff, G., Aran, D., Kark, J.D., Rosenberg, M., Dubnikov, T., Nissan, B., Wainstein, J., Friedlander, Y., Levy-Lahad, E., Glaser, B. and Hellman, A. (2012) Genome-wide survey reveals predisposing diabetes type 2-related DNA methylation variations in human peripheral blood. Human Molecular Genetics, 21, 371-383. doi:10.1093/hmg/ddr472 
[20] Ahmad, T., Lee, I.M., Pare, G., Chasman, D.I., Rose, L., Ridker, P.M. and Mora, S. (2011) Lifestyle interaction with fat mass and obesity-associated (FTO) genotype and risk of obesity in apparently healthy US women. Diabetes Care, 34, 675-680. doi:10.2337/dc10-0948

[21] Vimaleswaran, K.S., et al. (2012) Association between FTO variant and change in body weight and its interac- tion with dietary factors: The DiOGenes study. Obesity, 20, 1669-1674. doi:10.1038/oby.2012.49

[22] Galbete, C., Toledo, J., Martinez-Gonzalez, M.A., Martinez, J.A., Guillen-Grima, F. and Marti, A. (2012) Lifestyle factors modify obesity risk linked to PPARG2 and FTO variants in an elderly population: A cross-sectional analysis in the SUN Project. Genes Nutrition, 8, 61-67. 